\title{
Meta-analysis examining overall survival in patients with pancreatic cancer treated with second-line 5-fluorouracil and oxaliplatin-based therapy after failing first- line gemcitabine-containing therapy: effect of performance status and comparison with other regimens
}

Zev A. Wainberg ${ }^{1 *}$, Kynan Feeney ${ }^{2}$, Myung Ah Lee ${ }^{3}$, Andrés Muñoz ${ }^{4}$, Antonio Cubillo Gracián ${ }^{5,6}$, Sara Lonardi ${ }^{7}$, Baek-Yeol Ryoo ${ }^{8}$, Annie Hung ${ }^{9}$, Yong Lin ${ }^{10}$, Johanna Bendell ${ }^{11}$ and J. Randolph Hecht ${ }^{1}$

\begin{abstract}
Background: Pancreatic cancer has a poor prognosis and few choices of therapy. For patients with adequate performance status, FOLFIRINOX or gemcitabine plus nab-paclitaxel are preferred first-line treatment. 5-Fluorouracil (5-FU)-based therapy (e.g. FOLFIRI, OFF, or FOLFOX) are often used in patients who previously received gemcitabine-based regimens. A systematic review was conducted of the safety and efficacy of FOLFOX for metastatic pancreatic cancer following prior gemcitabine-based therapy. A Bayesian fixed-effect meta-analysis with adjustment of patient performance status (PS) was conducted to evaluate overall survival (OS) and compare outcomes with nanoliposomal irinotecan combination therapy.

Methods: PubMed.gov, FDA.gov, ClinicalTrials.gov, congress abstracts, Cochrane.org library, and EMBASE database searches were conducted to identify randomized controlled trials of advanced/metastatic disease, prior gemcitabine-based therapy, and second-line treatment with 5-FU and oxaliplatin. The database search dates were January 1, 1990-June 30, 2019. Endpoints were OS and severe treatment-related adverse events (TRAEs). Trial-level PS scores were standardized by converting Karnofsky grade scores to Eastern Cooperative Oncology Group (ECOG) Grade, and overall study-weighted PS was calculated based on weighted average of all patients.

\footnotetext{
* Correspondence: zwainberg@mednet.ucla.edu

'Department of Medicine, Division of Hematology/Oncology, David Geffen

School of Medicine, University of California Los Angeles, Los Angeles, CA,

USA

Full list of author information is available at the end of the article
}

(c) The Author(s). 2020 Open Access This article is licensed under a Creative Commons Attribution 4.0 International License, which permits use, sharing, adaptation, distribution and reproduction in any medium or format, as long as you give appropriate credit to the original author(s) and the source, provide a link to the Creative Commons licence, and indicate if changes were made. The images or other third party material in this article are included in the article's Creative Commons licence, unless indicated otherwise in a credit line to the material. If material is not included in the article's Creative Commons licence and your intended use is not permitted by statutory regulation or exceeds the permitted use, you will need to obtain permission directly from the copyright holder. To view a copy of this licence, visit http://creativecommons.org/licenses/by/4.0/ The Creative Commons Public Domain Dedication waiver (http://creativecommons.org/publicdomain/zero/1.0/) applies to the data made available in this article, unless otherwise stated in a credit line to the data. 
(Continued from previous page)

Results: Of 282 studies identified, 11 randomized controlled trials $(N=454)$ were included in the meta-analysis. Baseline weighted PS scores predicted OS in 10 of the 11 studies, and calculated PS scores of 1.0 were associated with a median OS of 6.3 months (95\% posterior interval, 5.4-7.4). After adjusting for baseline PS, FOLFOX had a similar treatment effect profile (median OS, range 2.6-6.7 months) as 5-FU/leucovorin plus nanoliposomal irinotecan therapy (median OS, 6.1 months; 95\% confidence interval 4.8-8.9). Neutropenia and fatigue were the most commonly reported Grade 3-4 TRAEs associated with FOLFOX.

Conclusions: Baseline PS is a strong prognostic factor when interpreting the efficacy of 5-FU and oxaliplatin-based therapy of pancreatic cancer after progression on first-line gemcitabine-based regimens. When baseline PS is considered, FOLFOX has a similar treatment effect as 5-FU and nanoliposomal irinotecan therapy and a comparable safety profile. These findings suggest that 5-FU and oxaliplatin-based therapies remain an acceptable and alternative second-line treatment option for patients with pancreatic cancer and adequate PS (e.g. ECOG 0-1) following gemcitabine treatment.

Keywords: Pancreatic cancer, Metastatic, Performance status, FOLFOX, Meta-analysis

\section{Background}

Pancreatic cancer is the seventh leading cause of global cancer death [1] and the third most common cause of cancer-related death in the United States [2]. It is usually diagnosed at an advanced stage, and $80-90 \%$ of patients with pancreatic cancer have unresectable tumors. For patients with metastatic disease, the 5-year survival rate is less than $10 \%$ [3]. The National Comprehensive Cancer Network (NCCN) 2019 guidelines recommend chemotherapy with FOLFIRINOX [4] or gemcitabine plus nab-paclitaxel [5] as preferred options for patients with an acceptable baseline performance status (Eastern Cooperative Oncology Group performance status [ECOG PS] score of 0-1) [6]. Cell-autonomous mechanisms of resistance to chemotherapy, however, further limit therapeutic options, and there have been multiple negative randomized trials in the adjuvant and first-line setting [7]. Immunotherapies explored so far have not demonstrated improved benefits over chemotherapy perhaps because tumor cells are nonimmunogenic in nature and are characterized by poor antigenicity [8]. Only $1 \%$ of patients with pancreatic cancer have tumors with high levels of microsatellite instability (MSI-H) or mismatch repair deficiencies (dMMR) and are considered to be candidates for checkpoint inhibitors $[9,10]$. Furthermore, in the small minority of patients with pancreatic cancer who have germline BRCA mutations (4-7\%), progression-free survival (PFS) following poly(adenosine diphosphate-ribose) polymerase (PARP) inhibitor therapy was not influenced by prior response to platinumbased therapy [11].

In general, most guidelines recommend the use of gemcitabine as monotherapy or as part of a combination therapy regimen for patients previously treated with FOLFIRINOX or other fluoropyrimidine-based therapy [6]. For patients previously treated with gemcitabinebased regimens, 5-FU-based therapy including FOLFIRI,
OFF, and FOLFOX has been recommended [6]. Recently, the Food and Drug Administration (FDA) approved nanoliposomal irinotecan in combination with 5-FU and leucovorin as second-line therapy after previous gemcitabine-based therapy (NAPOLI-1) [12]. Based on the findings from the NAPOLI-1 study, updated guidelines recommend the use of nanoliposomal irinotecan with fluorouracil and leucovorin in patients with metastatic pancreatic cancer after prior gemcitabinebased therapy [13]. In the NAPOLI-1 study, the median overall survival (OS) was 6.1 months (95\% confidence interval [CI] 4.8-8.9) for the combination of nanoliposomal irinotecan/5-FU/leucovorin compared with 4.2 months (95\% CI 3.3-5.3) for 5-FU/leucovorin alone with a hazard ratio of $0.67(95 \% \mathrm{CI} 0.49-0.92 ; P=.012)$ in patients with Karnofsky PS scores of 70 and above [12]. Survival benefits of this regimen were numerically similar to historically 5-FU-based therapy. For example, the phase III CONKO-003 trial of OFF demonstrated a median OS of 5.9 months [14]. More recently, a randomized phase II trial of mFOLFOX reported a median OS of 6.7 months in patients previously treated with gemcitabine [15], and despite not meeting its primary endpoint, the phase III PANCREOX study of mFOLFOX demonstrated a median OS of 6.1 months [16].

In the past, many prognostic factors have been identified and considered, such as hemoglobin level, tumor burden, liver metastases, venous thromboembolism, baseline expression of $\mathrm{B} 7 \mathrm{H} 1$ or $\mathrm{B} 7 \mathrm{H} 4$, and baseline CA19-9 [17-23]. One of the most significant prognostic factors is baseline ECOG PS. For example, one small, single-arm, phase II cohort study demonstrated a median OS for second-line FOLFOX with a median survival of 4.3 months. When patients were stratified by baseline ECOG PS, the median OS was 5.9 months for patients with adequate PS (i.e., ECOG PS scores, 0-1) and 2.6 months for those with ECOG PS scores $\geq 2$ [24]. In this 
paper, we performed a systematic review to better characterize the safety and efficacy of FOLFOX treatment for patients with metastatic pancreatic cancer following prior gemcitabine-based therapy. A Bayesian meta-analysis with adjustment of patient PS was conducted to evaluate the median OS and cross-compare with nanoliposomal irinotecan combination therapy.

\section{Methods}

\section{Literature search}

Studies were identified from searches conducted in PubMed.gov, FDA.gov, ClinicalTrials.gov, abstracts from individual congress proceedings, the Cochrane.org library, and the EMBASE database between January 1, 1990 and June 30, 2019. The search terms used were "pancreatic cancer", "gemcitabine", "FOLFOX", 5fluorouracil", "oxaliplatin", and "leucovorin".

\section{Inclusion and exclusion criteria}

Trials meeting the following criteria were included in the meta-analysis: 1) patients with locally advanced and metastatic disease, 2) patients who received prior gemcitabine-containing treatment, 3) second-line treatment regimens included 5-FU and oxaliplatin, and 4) reported data included median OS, severe (Grades 3-4) treatment-related adverse events (TRAEs), based on the Common Terminology Criteria for Adverse Events (CTCAE) v4.0 [25]. Trials meeting the following criteria were excluded from the metaanalysis: 1) patients who received prior treatment with 5-FU and oxaliplatin for locally advanced or metastatic pancreatic cancer, 2) patients who received an oral fluoropyrimidine, or irinotecan, capecitabine, or cisplatin as second-line treatment, and 3) patient PS was not reported.

\section{Data collection and analysis}

Two reviewers independently evaluated the literature identified from the database searches. For studies reported in different publications, the most recent study was retained, and the other version was excluded. The information extracted from each study included author names, publication year, number of patients, number of survival events, median OS, and severe adverse events. Any discrepancies in study eligibility or data extraction were reconciled. Studies were excluded if the full text of the publication was not available or if PS or median OS data were not reported.

\section{Statistical analyses}

The primary endpoint and secondary endpoints were median OS and severe TRAEs for patients who received FOLFOX or 5-FU/oxaliplatin-based therapy

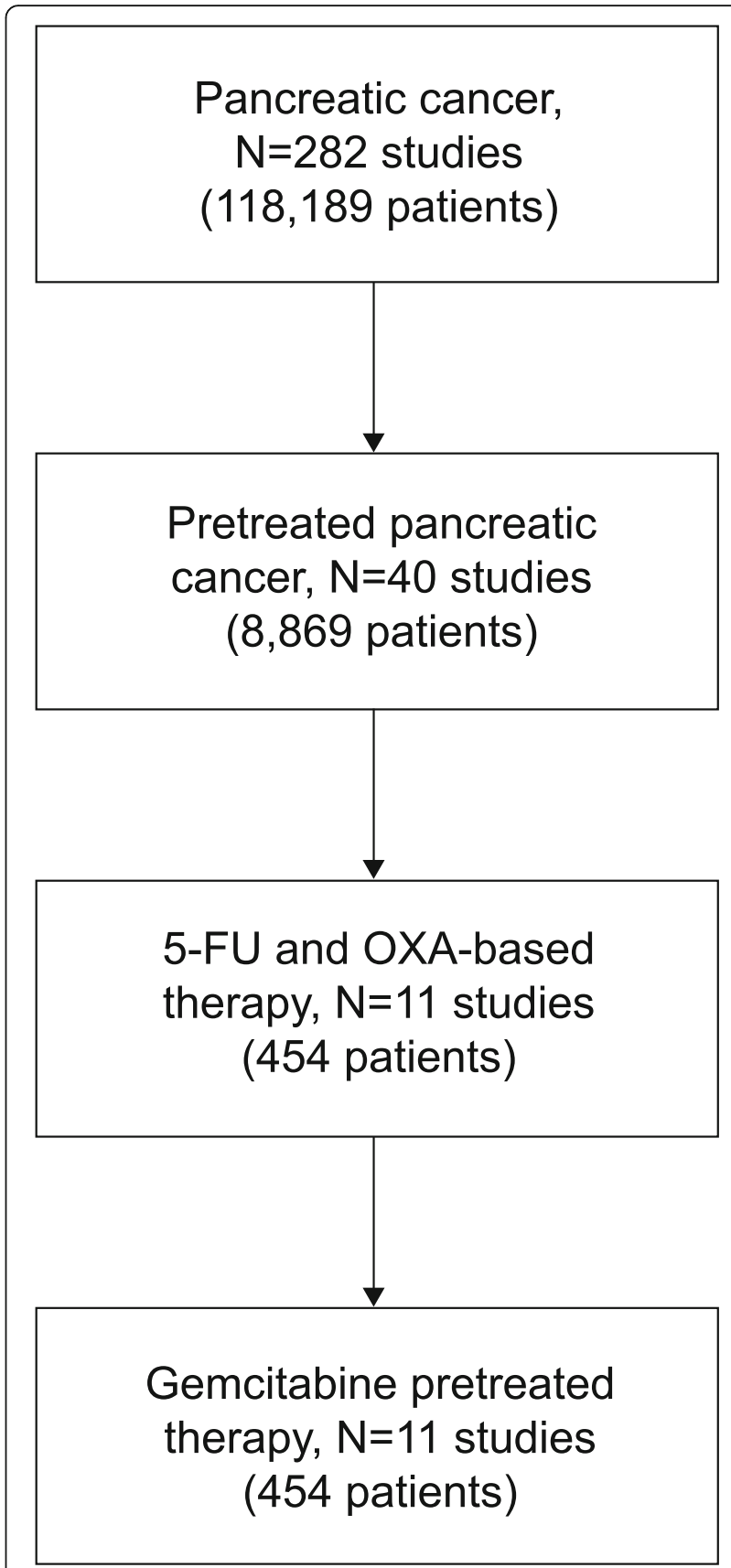

Fig. 1 CONSORT diagram

following prior gemcitabine-based regimens for metastatic pancreatic cancer. Adjusted PS was included in the meta-analysis model as follows. To standardize the trial-level PS, Karnofsky grade was converted to ECOG Grade according to Oken et al. [26], and the overall study-weighted PS was calculated based on the weighted average. For example, ECOG $0-1$ was converted to numerical value 1 , and ECOG 2,3 , and 4 were converted to numerical values 2,3 , and 4 , respectively. For a study with $w_{1} \%$ of the patients had 
Table 1 Summary of 5-FU and oxaliplatin-based therapy as second-line therapy

\begin{tabular}{|c|c|c|c|c|c|c|c|c|}
\hline Treatment & Author/year & $N$ & Weighted PS & Original PS & Prior surgery $(\%)$ & Deaths & Median OS (m) & ORR (\%) \\
\hline OFF & Pelzer 2009 [28] & 37 & 1.5 & KS: Median: 70, range: $[60,90]$ & 43 & 33 & 5.1 & 6 \\
\hline OFF & Pelzer 2011 [27] & 23 & 1.3 & KS: Median: 80, range: $[70,100]$ & NR & 18 & 4.8 & 0 \\
\hline OFF & Oettle 2014 [14] & 76 & 1.2 & KS: (90-100) (53.9\%), 70-80 (46.1\%) & 45 & 73 & 5.9 & 17 \\
\hline 5-FU/OXA-based & Tsavaris 2005 [29] & 30 & 1.7 & KS: (80-100) (33.4\%), 70-50 (66.7\%) & NR & 20 & 5.7 & 23 \\
\hline FOLFOX & Gebbia 2007 [30] & 42 & 1.4 & ECOG: 1 (62\%), 2 (38\%) & 9 & 38 & 6.7 & 14 \\
\hline FOLFOX & Yoo 2009 [31] & 30 & 1.0 & ECOG: 0-1 (97\%) & 32 & 25 & 3.8 & 7 \\
\hline FOLFOX & Zaanan 2014 [24] & 12 & 1.0 & ECOG: 0-1 (100\%) & 0 & 10 & 5.9 & 0 \\
\hline FOLFOX & Zaanan 2014 [24] & 12 & 2.5 & ECOG: $2-3(100 \%)$ & 0 & 12 & 2.6 & 0 \\
\hline FOLFOX & Gill 2016 [16] & 54 & 1.1 & ECOG: 0-1 (89\%), 2 (11\%) & NR & 47 & 6.1 & 13 \\
\hline FOLFOX & Berk 2012 [32] & 46 & 1.2 & ECOG: 0-1 (78\%), 2 (22\%) & NR & 33.6 & 6.2 & 17 \\
\hline OFF & El-Hadaad 2013 [33] & 30 & 1.2 & ECOG: $0-1$ (83.4\%), 2 (16\%) & NR & 29 & 5.1 & 7 \\
\hline FOLFOX & Chung 2017 [15] & 62 & 1.0 & ECOG: 0-1 (100\%) & NR & 53 & 6.7 & 11 \\
\hline
\end{tabular}

Abbreviations: 5-FU 5-fluorouracil, ECOG Eastern Cooperative Oncology Group, FOLFOX leucovorin/5-fluorouracil/oxaliplatin, $m$ months, KS Karnofsky status, NR not reported, OFF oxaliplatin/5-fluorouracil/leucovorin, ORR overall response rate, OS overall survival, OXA oxaliplatin, PS performance score

ECOG $0-1$ and $w_{2} \%$ with ECOG 2. The weighted trial performance was calculated as: $1 \times w_{1} \%+2 \times$ $w_{2} \%$. A Bayesian fixed-effect meta-analysis was performed for the median OS with weighted trial PS as a predictor. A noninformative prior was used to establish the relationship between log transformation of median OS and PS. The noninformative prior was assumed for the related parameters. The posterior median of OS and $95 \%$ posterior interval (PI) were summarized for patients with ECOG PS $\leq 1$. For safety, Grade 3/4 clinically relevant toxicities that were reported in $\geq 10 \%$ of patients in any trial were pooled together to evaluate the toxicity of the treatment regimen. To be conservative, trials that did not report a specific adverse event were removed from the group of evaluable patients. All analyses were performed in R 3.5.0.

\section{Results}

\section{Study selection}

The CONSORT flow chart that illustrates study identification and selection for the meta-analysis is shown in Fig. 1. Of 282 studies identified in the database searches, 11 were chosen for meta-analysis [14-16, 24, 27-33], and 242 studies were excluded. In total, 454 patients with pancreatic cancer were included in this meta-analysis. The 11 selected studies evaluated 5-FU and oxaliplatin-based

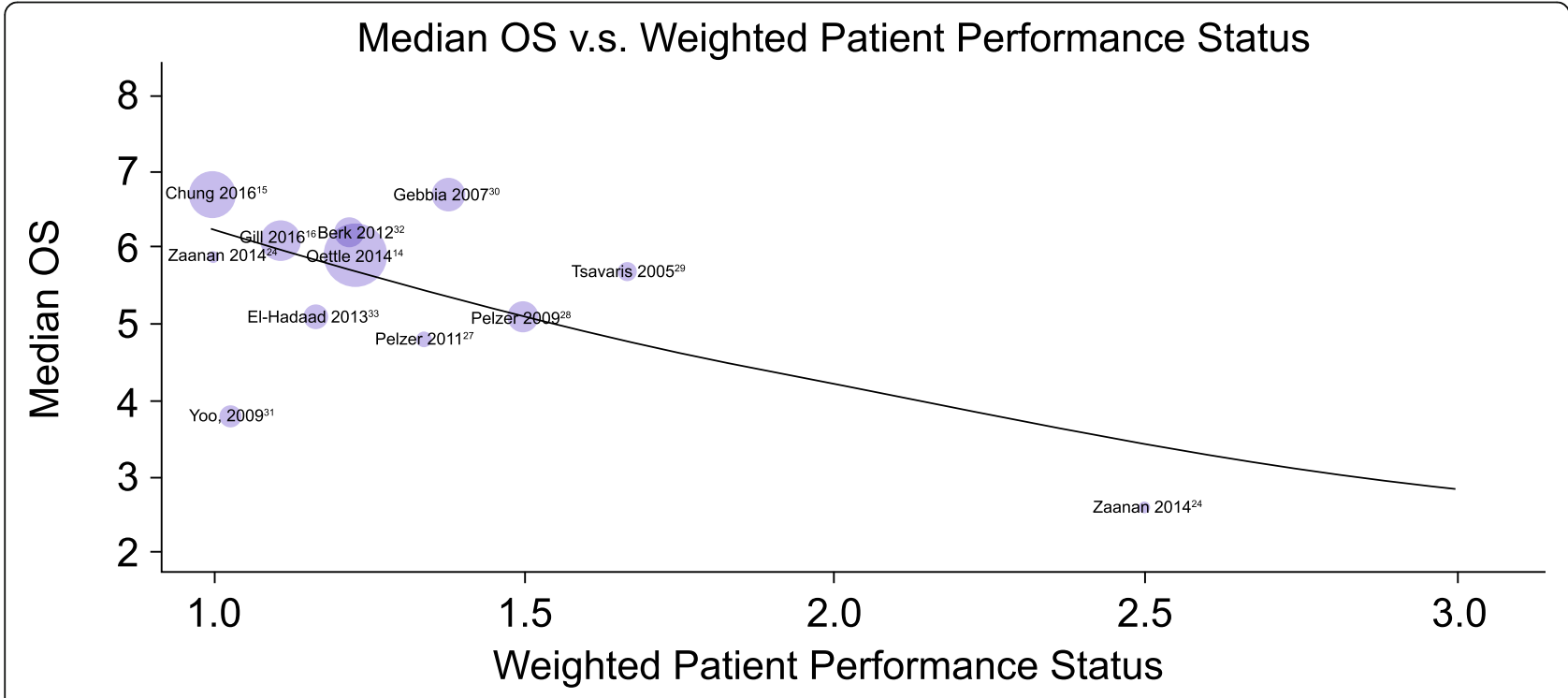

Fig. 2 Association between median overall survival (OS) and patient performance status 


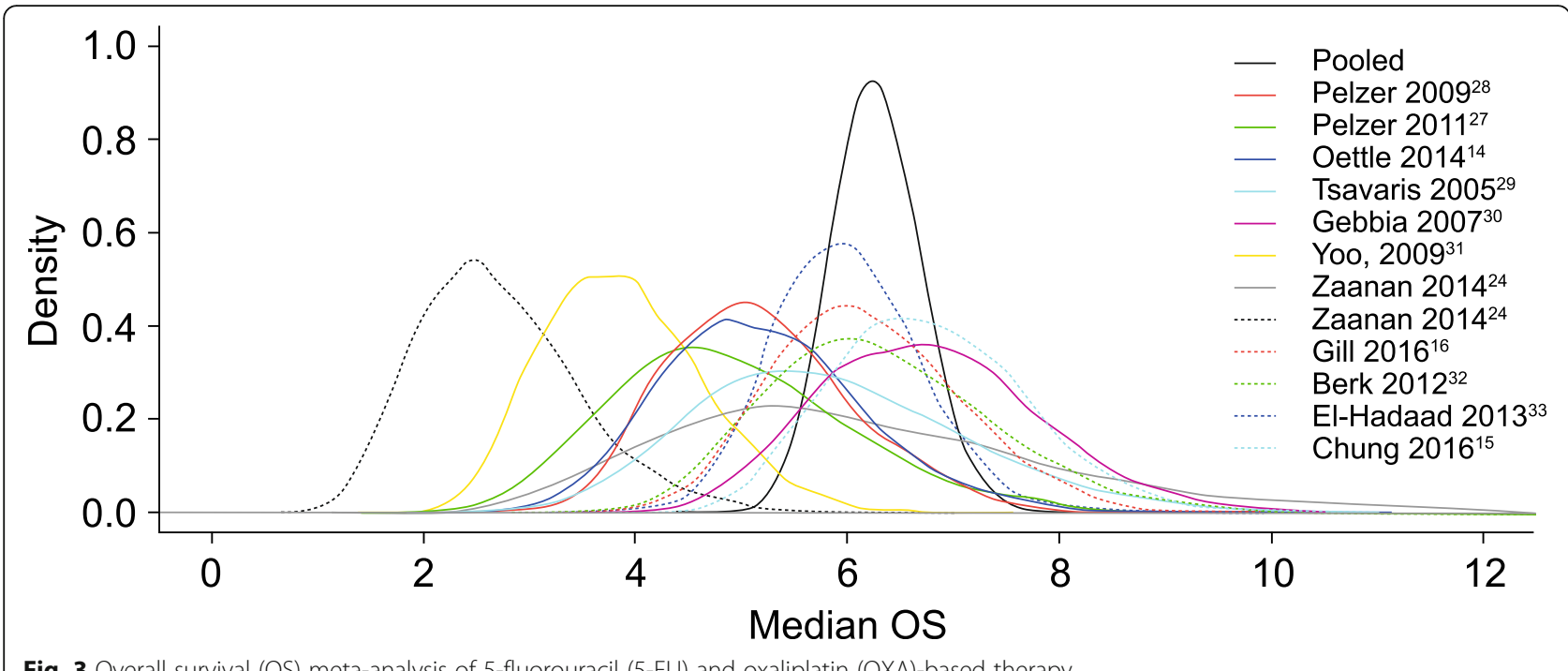

Fig. 3 Overall survival (OS) meta-analysis of 5-fluorouracil (5-FU) and oxaliplatin (OXA)-based therapy

regimens, including OFF, FOLFOX, and modified FOLFOX (mFOLFOX6, mFOLFOX4) (Table 1).

\section{Patient population}

In the 454 evaluable patients, the reported PS ranged from Karnofsky performance index scores of 60-100 and ECOG PS scale scores of 0-3 (Table 1). Of 11 studies, five reported the surgical histories of the patient sample. Rates of prior surgery were $8 \%$ [34], 9\% [30], $32 \%$ [31], 43\% [28], and 45\% [14]. The median OS ranged from 2.6 months to 6.7 months, and the overall response rate ranged from 0 to $23 \%$ (Table 1).

\section{Overall survival}

Baseline weighted PS scores predicted OS in 10 of the 11 studies (Fig. 2). Results from one study were identified as an outlier, with a median OS of approximately 4 months in patients with a baseline weighted PS score of 1.0 [31]. Likely the variability was because of a long period of time between the conclusion of gemcitabine-based therapy to FOLFOX treatment (median 15 weeks, range 7.0-32.6 weeks). To maintain integrity of the analysis, the outlier was not removed from the model. Based on the Bayesian meta-analysis with the adjustment of baseline PS, for 5-FU and oxaliplatin-based therapy (Fig. 3), the median OS was 6.2 months (95\% PI 5.4-7.1). For the analysis of FOLFOX therapy (Fig. 4), the median OS was 6.3 months (95\% PI 5.4-7.4).

\section{Safety of FOLFOX}

The clinically relevant Grade 3-4 TRAEs for the selected studies were pooled, and the results are summarized in

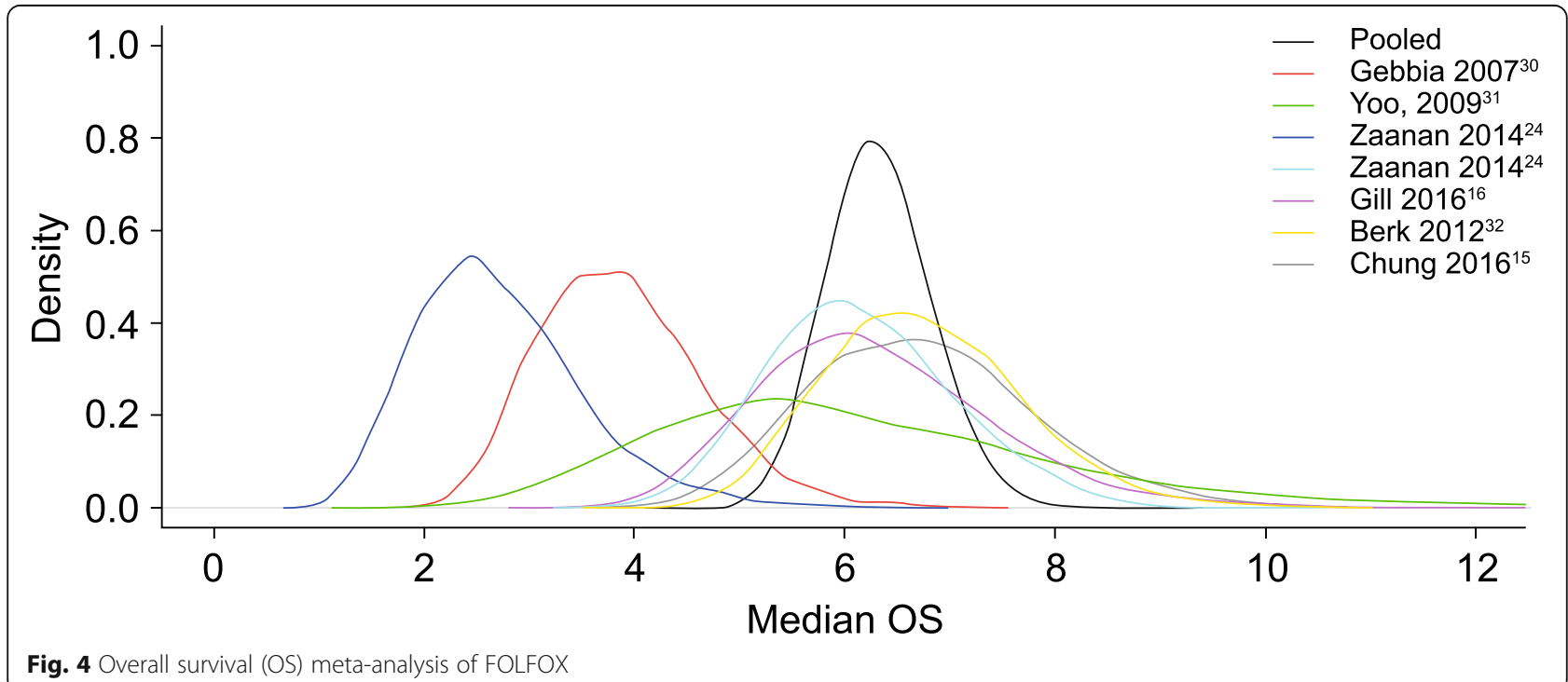


Table 2. The most commonly reported Grade 3-4 TRAEs associated with FOLFOX therapy were neutropenia (21.5\%) and fatigue (11.7\%). Other Grade 3-4 TRAEs occurring in $>10 \%$ in any trial were neurotoxicity (5.3\%), thrombocytopenia (4.9\%), anemia (4.5\%), diarrhea (4.2\%), and vomiting (4.1\%).

\section{Discussion}

The prognosis of pancreatic cancer remains dismal, and the primary first-line treatments for patients with metastatic disease are gemcitabine-based combinations and FOLFIRINOX. For patients previously treated with gemcitabine, second-line 5-FU-based therapy including FOLFIRI, FOLFOX, and OFF have been recommended [6]. In randomized trials, oxaliplatin-based regimens in the second-line setting, such as CONKO-003 and PANCREOX, have had conflicting efficacy results [35]. In the CONKO-003 trial, the OFF regimen was superior to FF (leucovorin and 5-FU) with a median OS of 5.9 vs. 3.3 months, respectively [14]. On the other hand, the PANCREOX study compared a different oxaliplatin, 5-FU, and leucovorin-containing regimen (mFOLFOX6) with 5 -FU/LV, with a median OS of 6.1 vs. 9.9 months, respectively [16]. While mFOLFOX6 produced results consistent with prior studies of oxaliplatin and 5-FU combinations, the 5-FU/LV control arm demonstrated surprisingly prolonged survival. One factor that may have contributed to these findings was an imbalance in several baseline characteristics. For example, the median time from diagnosis of advanced disease to treatment was longer in the mFOLFOX6 arm compared with the 5-FU/LV arm (7.9 vs. 5.7 months, respectively), and a higher proportion of patients in the mFOLFOX6 arm than in the 5-FU/LV arm had baseline ECOG PS scores of $2(11.1 \%$ vs. $5.7 \%)$. Additionally, fewer patients in the mFOLFOX6 arm than the 5-FU/LV arm received postdiscontinuation therapy ( $7 \%$ vs. $23 \%$, respectively). It is important to remember that these are relatively small studies of fewer than 200 patients each, and comparisons are fraught because of inherent methodologic differences.

The systematic literature review and meta-analysis reported here was conducted in an attempt to overcome the variability induced by small sample sizes. In addition, after adjusting for PS, the meta-analysis of 5-FU and oxaliplatin-based therapy (e.g., FOLFOX) demonstrated a numerically similar treatment effect (median OS range 2.6-6.7 months; Table 1) compared with 5-FU/LV plus nanoliposomal irinotecan combination therapy in the NAPOLI-1 trial (median OS 6.1 months; 95\% CI 4.88.9) (Table 3) [12]. For patients with ECOG PS of 0 or 1 , the median OS was 6.2 months (95\% PI 5.4-7.1) for patients who received the oxaliplatin, 5-FU, and LV regimen. In addition, for the subset meta-analysis of FOLFOX therapy (Fig. 4), the median OS demonstrated consistent results with median OS of 6.3 months (95\% PI 5.4-7.4). The most commonly reported Grade 3-4 TRAEs associated with FOLFOX therapy were neutropenia (21.5\%) and fatigue (11.7\%). Other Grade 3-4 TRAEs occurring in $>10 \%$ in any trial were neurotoxicity (5.3\%), thrombocytopenia (4.9\%), anemia (4.5\%), diarrhea (4.2\%), and vomiting (4.1\%) (Table 2). Based on an indirect comparison, this adverse event profile was similar to the findings of the NAPOLI-1 trial (Table 4).

Table 2 Summary of safety for 5-FU and oxaliplatin-based therapy

\begin{tabular}{|c|c|c|c|c|c|c|c|c|c|}
\hline \multirow[t]{2}{*}{ Treatment } & \multirow[t]{2}{*}{ Author/year } & \multirow[t]{2}{*}{$N$} & \multicolumn{7}{|c|}{ Grade $3-4$ clinically relevant toxicities $>10 \%$ in any trial } \\
\hline & & & Diarrhea & Neutropenia & Anemia & Neurotoxicity & Fatigue $^{a}$ & Vomiting & Thrombocytopenia \\
\hline OFF & Pelzer 2009 [28] & 37 & 8.1 & NR & NR & 10.8 & NR & 13.5 & 0 \\
\hline OFF & Pelzer 2011 [27] & 23 & 8.7 & NR & NR & NR & NR & NR & NR \\
\hline OFF & Oettle 2014 [14] & 76 & 1.3 & NR & 3.9 & NR & NR & 1.3 & 1.3 \\
\hline 5-FU/OXA-based & Tsavaris 2005 [29] & 30 & 14.2 & NR & 3.2 & 4.2 & 0 & 0 & 3.2 \\
\hline FOLFOX & Gebbia 2007 [30] & 42 & NR & 17 & 14 & 12 & NR & NR & 7 \\
\hline FOLFOX & Yoo 2009 [31] & 30 & 0 & 20 & 3 & 0 & 14 & 10 & 3 \\
\hline FOLFOX & Zaanan 2014 [24] & 27 & 0 & 7.4 & 7.4 & 7.4 & 14.8 & 0 & 11.1 \\
\hline FOLFOX & Gill 2016 [16] & 49 & 2 & 32.7 & 2 & 4.1 & 14.2 & 4.1 & 8.2 \\
\hline FOLFOX & Berk 2012 [32] & 46 & 2 & 22 & 0 & NR & NR & 2 & 7 \\
\hline OFF & El-Hadaad 2013 [33] & 30 & 3.3 & 23.2 & 6.6 & 6.6 & NR & 3.3 & 6.6 \\
\hline FOLFOX & Chung 2017 [15] & 62 & 6.5 & NR & 3.2 & 0 & 12.9 & 4.8 & NR \\
\hline \multicolumn{3}{|c|}{ Evaluable patients for each $A E, n$} & 410 & 224 & 392 & 307 & 198 & 387 & 367 \\
\hline \multicolumn{3}{|c|}{ Weighted average (\%) } & 4.2 & 21.5 & 4.5 & 5.3 & 11.7 & 4.1 & 4.9 \\
\hline
\end{tabular}

Abbreviations: 5-FU 5-fluorouracil, AE adverse event, FOLFOX leucovorin/5-fluorouracil/oxaliplatin, $N$ patients in each study, $n$ evaluable patients for each AE, NR not reported, OFF oxaliplatin/5-fluorouracil/leucovorin, OXA oxaliplatin

${ }^{a}$ Fatigue includes reported terms of fatigue and asthenia 
Table 3 Baseline and efficacy profile for nanoliposomal irinotecan-based therapy from NAPOLI-1 [12]

\begin{tabular}{ll}
\hline Characteristics $^{\mathrm{a}}$ & Results \\
\hline$N$ & 117 \\
Karnofsky performance 100-80 & $91 \%$ \\
Lines of prior therapy: 0/1/2+ (\%) & $13 / 53 / 34$ \\
Prior therapy: & $45 / 55 / 43$ \\
Gemcitabine mono/combination/5-FU-based (\%) &
\end{tabular}

Median OS $(95 \% \mathrm{Cl})$

6.1 months (4.8-8.9)

Abbreviations: 5-FU 5-fluorouracil, Cl confidence interval, ECOG Eastern Cooperative Oncology Group, $N$ patients in study, OS overall survival ${ }^{\text {a }}$ For patients with ECOG $0-1$, the poster median of the median OS for 5-FU and oxaliplatin-based therapy and FOLFOX in second-line are 6.2 months and 6.3 months, respectively

These analyses are not without limitations. Our ability to adjust survival outcomes for other potential prognostic factors was hindered because we did not have access to the full study datasets. For example, prior surgery, levels of the CA-19-9 antigen, baseline hemoglobin levels, $B R C A 1$ or $B R C A 2$ mutation status, or the time from diagnosis to the initiation of treatment were not always reported. In addition, the cross-trial comparison between the meta-analysis of the FOLFOX treatment regimen and the results from NAPOLI-1 are indirect and must be interpreted with caution.

\section{Conclusions}

In this meta-analysis, we confirmed that baseline PS is a strong prognostic factor when interpreting the efficacy of 5-FU and oxaliplatin-based therapy after progression of pancreatic cancer with first-line gemcitabinecontaining therapies. After adjusting for patient PS, the meta-analysis of 5-FU and oxaliplatin-based therapy (e.g., FOLFOX) shows a numerically similar treatment effect as 5-FU and nanoliposomal irinotecan therapy in the NAPOLI-1 trial. In addition, the adverse event profile is also comparable between the two treatment

Table 4 Safety profile for nanoliposomal irinotecan-based therapy [12]

\begin{tabular}{lll}
\hline Grade 3-4 AEs & $\begin{array}{l}\text { Nanoliposomal irinotecan- } \\
\text { based therapy (\%) }\end{array}$ & $\begin{array}{l}\text { 5-FU and OXA-based } \\
\text { therapy weighted } \\
\text { average (\%) [range }]\end{array}$ \\
\hline Diarrhea & 13 & $4.2[0,14.2]$ \\
Vomiting & 11 & $4.1[0,13.5]$ \\
Fatigue & 14 & $11.7[0,14.8]$ \\
Neutropenia & 27 & $21.5[7.4,32.7]$ \\
Anemia & 9 & $4.5[0,14]$ \\
Hypokalemia & 3 & $\mathrm{NR}$ \\
Neurotoxicity & $\mathrm{NR}$ & $5.3[0,12]$ \\
Thrombocytopenia & $\mathrm{NR}$ & $4.9[0,11.1]$ \\
\hline
\end{tabular}

Abbreviations: 5-FU 5-fluorouracil, $A E$ adverse event, $N R$ not recorded, OXA oxaliplatin regimens. The findings from our analyses suggest that the combination of 5-FU and oxaliplatin-based therapies remains an acceptable and alternative second-line treatment option for patients with pancreatic cancer and adequate PS (e.g., ECOG 0/1) who have received gemcitabine-based therapies.

\section{Abbreviations}

Cl: Confidence interval; CTCAE: Common Terminology Criteria for Adverse Events; dMMR: Mismatch repair deficiencies; ECOG: Eastern Cooperative Oncology Group; FDA: Food and Drug Administration; 5-FU: 5-fluorouracil; MSI-H: High level of microsatellite instability; NCCN: National Comprehensive Cancer Network; OS: Overall survival; PARP: Poly(adenosine diphosphateribose) polymerase; PFS: Progression-free survival; PI: Posterior interval; PS: Performance status; TRAE: Treatment-related adverse event

\section{Acknowledgements}

The authors thank David Ferry and Sujata Rao for their thoughtful review of the manuscript. Medical writing assistance was provided by Sally Laden of ProScribe Medical Affairs - Envision Pharma Group, and was funded by Eli Lilly and Company. ProScribe Medical Affairs' services complied with international guidelines for Good Publication Practice (GPP3).

\section{Authors' contributions}

ZAW, B-YR, M-AL, AH, and YL participated in the meta-analysis methodology and design; $Y L$ and $A H$ screened identified literature and conducted data extraction and statistical analysis. ZAW, KF, M-AL, AM, ACG, SL, B-YR, AH, YL, JB and $J R H$ were involved in the interpretation of the data. $Y L$ wrote the manuscript. ZAW, KF, M-AL, AM, ACG, SL, B-YR, AH, YL, JB and JRH contributed to improving the manuscript and read and approved the version of the manuscript to be published. All authors take responsibility for appropriate content.

\section{Funding}

Eli Lilly and Company was involved in the study design, data collection, data analysis, and preparation of the manuscript.

\section{Availability of data and materials}

All data generated or analyzed during this study are available from the publications cited in the reference list.

Ethics approval and consent to participate

Not applicable.

\section{Consent for publication}

Not applicable.

\section{Competing interests}

ZAW has received research funding from AstraZeneca, Bristol-Myers Squibb, Eli Lilly and Company, Ipsen, and Merck, and has served as a consultant and on advisory panels for Bayer, Eli Lilly and Company, Daiichi Sankyo, EMD Serono, Five Prime, Ipsen, Macrogenics, Merck, Molecular Templates, and Novartis. AM has received research funding from Leo Pharma and Sanofi; has served as a consultant and on advisory panels for Bristol-Myers Squibb, Celgene, Daiichi Sankyo, Halozyme, Leo Pharma, Pfizer, and Sanofi; has participated in speaker bureaus for Bayer, Eli Lilly and Company, MSD, Rovi, and Servier; has received reimbursements for travel, accommodations, and expenses from Celgene and Roche; and holds patents, royalties, and other intellectual property (risk assessment model in venous thromboembolism in patients with cancer). SL has received research funding from Amgen and Merck Serono; has served as a consultant and on advisory panels for Amgen, Eli Lilly and Company, Merck Serono, and Servier; and has participated in speaker bureaus for Bristol-Myers Squibb, Eli Lilly and Company, Merck Serono, Roche, and Servier. JB's institution has received research funding from AbbVie, Acerta Pharma, ADC, Agios, Amgen, Apexigen, Arch Oncology, Arcus Biosciences, ARMO, Array, Arrys, AstraZeneca, Bayer, Bellicum, Blueprint, Boehringer Ingelheim, Boston Biomedical, Bristol-Myers Squibb, Calithera, Celgene, Celldex, CytomX, Daiichi Sankyo, Effector, Eisai, Eli Lilly and Company, EMD Serono, Evelo, Five Prime, FORMA, Forty Seven, Genentech/Roche, Gilead, Gossamer Bio, GSK, Harpoon, ImClone, Incyte, Innate, Ipsen, Jacobio, Koltan, LEAP, Macrogenics, Marshall Edwards, Medlmmune, Merck, Merrimack, 
Mersana, Merus, Millennium, Nektar, Novartis, Novocare, OncoGenex, OncoMed, Onyx, Pfizer, Pieris, Prelude Oncology, Rgenix, Sanofi, Seattle Genetics, Shattuck Labs, Sierra, SynDevRx, Takeda, Tarveda, Tracon, Tyrogenex Taiho, TempestTx, TG Therapeutics, Unum Therapeutics, and Vyriad; has served as a consultant and on advisory panels for Agios, Amgen, Apexigen, Arch Oncology, ARMO, Array, AstraZeneca, Bayer, BeiGene, Boehringer Ingelheim, Bristol-Myers Squibb, Celgene, Continuum Clinical, Cyteir, Daiichi Sankyo, Eli Lilly and Company, Five Prime, FORMA, Genentech/Roche, Gilead, GSK, Incyte, Innate, Ipsen, Janssen, LEAP, MacroGenics, Medlmmune, Merck, Merrimack, Moderna Therapeutics, Molecular Partners, Novartis, OncoGenex, OncoMed, Phoenix Bio, Prelude Therapeutics, Sanofi, Seattle Genetics, Taiho, Tanabe Research Laboratories, TD2 (Translational Drug Development), TG Therapeutics, Tizona, Tolero, and Torque; and has received reimbursements for food, beverage, and travel from ARMO, Boehringer Ingelheim, BristolMyers Squibb, Celgene, Eli Lilly and Company, FORMA, Genentech/Roche, Gilead, Ipsen, Medlmmune, Merck, Novartis, OncoMed, OncoGenex, and Taiho. JRH received grants, personal fees, and non-financial support from ARMO BioSciences, a wholly owned subsidiary of Eli Lilly and Company, during the conduct of the study.

$\mathrm{AH}$ and $\mathrm{YL}$ are employees of Eli Lilly and Company, and $\mathrm{YL}$ acknowledges stock/equity ownership in Eli Lilly and Company. KF, M-AL, ACG, and B-YR have no competing interests to declare.

\section{Author details}

${ }^{1}$ Department of Medicine, Division of Hematology/Oncology, David Geffen School of Medicine, University of California Los Angeles, Los Angeles, CA, USA. ${ }^{2}$ Notre Dame University, Fremantle and Edith Cowan University Joondalup, Perth, Australia. ${ }^{3}$ Catholic University of Korea, Seoul, South Korea. ${ }^{4}$ Hospital General Universitario Gregorio Marañón, Madrid, Spain. ${ }^{5} \mathrm{HM}$ Universitario Sanchinarro, Centro Integral Oncológico Clara Campal HM-CIOCC, Madrid, Spain. 'Departamento de Ciencias Médicas Clínicas Universidad San Pablo CEU, Madrid, Spain. ${ }^{7}$ Istituto Oncologico Veneto IRCCS, Padova, Italy. ${ }^{8}$ Asan Medical Center, University of Ulsan College of Medicine, Seoul, South Korea. ${ }^{9}$ ARMO Biosciences, a wholly owned subsidiary of Eli Lilly and Company, Redwood City, CA, USA. ${ }^{10}$ Eli Lilly and Company, Indianapolis, IN, USA. ${ }^{11}$ Sarah Cannon Research Institute/Tennessee Oncology, Nashville, TN, USA.

\section{Received: 20 February 2020 Accepted: 25 June 2020} Published online: 08 July 2020

\section{References}

1. Bray F, Ferlay J, Soerjomataram I, Siegel RL, Torre LA, Jemal A. Global cancer statistics 2018: GLOBOCAN estimates of incidence and mortality worldwide for 36 cancers in 185 countries. CA Cancer J Clin. 2018;68(6):394-424.

2. National Cancer Institute. Common cancer types 2019. Available from: https://www.cancer.gov/types/common-cancers.

3. Rawla P, Sunkara T, Gaduputi V. Epidemiology of pancreatic cancer: global trends, etiology and risk factors. World J Oncol. 2019;10(1):10-27.

4. Conroy T, Desseigne F, Ychou M, Bouché O, Guimbaud R, Bécouarn Y, et al. FOLFIRINOX versus gemcitabine for metastatic pancreatic cancer. N Engl J Med. 2011:364(19):1817-25

5. Von Hoff DD, Ervin T, Arena FP, Chiorean EG, Infante J, Moore M, et al. Increased survival in pancreatic cancer with nab-paclitaxel plus gemcitabine. N Engl J Med. 2013:369(18):1691-703.

6. National Comprehensive Cancer Network. National Comprehensive Cancer Network (NCCN) pancreatic adenocarcinoma (v3.2019) 2019. Available from: https://www.nccn.org/professionals/physician_gls/pdf/pancreatic.pdf.

7. Oberstein PE, Olive KP. Pancreatic cancer: why is it so hard to treat? Ther Adv Gastroenterol. 2013;6(4):321-37

8. Torphy RJ, Zhu Y, Schulick RD. Immunotherapy for pancreatic cancer: barriers and breakthroughs. Ann Gastroenterol Surg. 2018;2(4):27481.

9. Mori T, Hamaya Y, Uotani T, Yamade M, Iwaizumi M, Furuta T, et al. Prevalence of elevated microsatellite alterations at selected tetranucleotide repeats in pancreatic ductal adenocarcinoma. PLoS One. 2018;13(12): e0208557.

10. Hu Zl, Shia J, Stadler ZK, Varghese AM, Capanu M, Salo-Mullen E, et al. Evaluating mismatch repair deficiency in pancreatic adenocarcinoma: challenges and recommendations. Clin Cancer Res. 2018;24(6):1326-36.
11. Golan T, Hammel P, Reni M, Van Cutsem E, Macarulla T, Hall MJ, et al. Maintenance olaparib for germline BRCA-mutated metastatic pancreatic cancer. N Engl J Med. 2019;381(4):317-27.

12. Wang-Gillam A, Li CP, Bodoky G, Dean A, Shan YS, Jameson G, et al. Nanoliposomal irinotecan with fluorouracil and folinic acid in metastatic pancreatic cancer after previous gemcitabine-based therapy (NAPOLI-1): a global, randomised, open-label, phase 3 trial. Lancet. 2016;387(10018):54557.

13. ESMO. eUpdate - Cancer of the pancreas treatment recommendations 2017. Available from: https://www.esmo.org/Guidelines/GastrointestinalCancers/Cancer-of-the-Pancreas/eUpdate-Treatment-Recommendations.

14. Oettle H, Riess H, Stieler JM, Heil G, Schwaner I, Seraphin J, et al. Second-line oxaliplatin, folinic acid, and fluorouracil versus folinic acid and fluorouracil alone for gemcitabine-refractory pancreatic cancer: outcomes from the CONKO-003 trial. J Clin Oncol. 2014;32(23):2423-9.

15. Chung V, McDonough S, Philip PA, Cardin D, Wang-Gillam A, Hui L, et al. Effect of selumetinib and MK-2206 vs oxaliplatin and fluorouracil in patients with metastatic pancreatic cancer after prior therapy: SWOG S1115 study randomized clinical trial. JAMA Oncol. 2017:3(4):516-22.

16. Gill S, Ko YJ, Cripps C, Beaudoin A, Dhesy-Thind S, Zulfiqar M, et al. PANCREOX: a randomized phase III study of fluorouracil/leucovorin with or without oxaliplatin for second-line advanced pancreatic cancer in patients who have received gemcitabine-based chemotherapy. J Clin Oncol. 2016; 34(32):3914-20.

17. Furukawa K, Uwagawa T, Iwase R, Haruki K, Fujiwara Y, Gocho T, et al. Prognostic factors of unresectable pancreatic cancer treated with nafamostat mesilate combined with gemcitabine chemotherapy. Anticancer Res. 2012;32(11):5121-6.

18. Zhang DX, Dai YD, Yuan SX, Tao L. Prognostic factors in patients with pancreatic cancer. Exp Ther Med. 2012;3(3):423-32.

19. Tas F, Sen F, Odabas H, Kilıc L, Keskin S, Yıldız I. Performance status of patients is the major prognostic factor at all stages of pancreatic cancer. Int J Clin Oncol. 2013;18(5):839-46

20. Bilici A. Prognostic factors related with survival in patients with pancreatic adenocarcinoma. World J Gastroenterol. 2014;20(31):10802-12.

21. Bittoni A, Pellei C, Lanese A, Giampieri R, D'Angelo A, Giglio E, et al. Prognostic factors in advanced pancreatic cancer patients receiving secondline chemotherapy: a single institution experience. Transl Cancer Res. 2018; 7(5):1190-8.

22. Boone BA, Zenati MS, Rieser C, Hamad A, Al-Abbas A, Zureikat AH, et al. Risk of venous thromboembolism for patients with pancreatic ductal adenocarcinoma undergoing preoperative chemotherapy followed by surgical resection. Ann Surg Oncol. 2019:26(5):1503-11.

23. Qi ZJ, Yu D, Chen CH, Jiang H, Li R, Kang YM. The prognostic value of B7H1 and $\mathrm{B} 7 \mathrm{H} 4$ expression in pancreatic cancer: a meta-analysis. Int J Biol Markers. 2019;34(4):373-80

24. Zaanan A, Trouilloud I, Markoutsaki T, Gauthier M, Dupont-Gossart AC, Lecomte T, et al. FOLFOX as second-line chemotherapy in patients with pretreated metastatic pancreatic cancer from the FIRGEM study. BMC Cancer. 2014;14:441.

25. U.S. Department of Health and Human Services. Common Terminology Criteria for Adverse Events (CTCAE) Version 4.0 2009. Available from: https:// evs.nci.nih.gov/ftp1/CTCAE/CTCAE 4.03/Archive/CTCAE 4.0 2009-05-29 QuickReference_8.5x11.pdf.

26. Oken MM, Creech RH, Tormey DC, Horton J, Davis TE, McFadden ET, et al. Toxicity and response criteria of the eastern cooperative oncology group. Am J Clin Oncol. 1982;5(6):649-55.

27. Pelzer U, Schwaner I, Stieler J, Adler M, Seraphin J, Dörken B, et al. Best supportive care (BSC) versus oxaliplatin, folinic acid and 5-fluorouracil (OFF) plus BSC in patients for second-line advanced pancreatic cancer: a phase IIIstudy from the German CONKO-study group. Eur J Cancer. 2011;47(11): 1676-81.

28. Pelzer U, Stieler J, Roll L, Hilbig A, Dörken B, Riess H, et al. Second-line therapy in refractory pancreatic cancer. Results of a phase II study. Onkologie. 2009;32(3):99-102.

29. Tsavaris N, Kosmas C, Skopelitis H, Gouveris P, Kopterides P, Loukeris D, et al Second-line treatment with oxaliplatin, leucovorin and 5 -fluorouracil in gemcitabine-pretreated advanced pancreatic cancer: a phase II study. Investig New Drugs. 2005;23(4):369-75.

30. Gebbia V, Maiello E, Giuliani F, Borsellino N, Caruso M, Di Maggio G, et al. Second-line chemotherapy in advanced pancreatic carcinoma: a multicenter 
survey of the Gruppo Oncologico Italia Meridionale on the activity and safety of the FOLFOX4 regimen in clinical practice. Ann Oncol. 2007; 18(Suppl 6):vi124-7.

31. Yoo C, Hwang JY, Kim JE, Kim TW, Lee JS, Park DH, et al. A randomised phase II study of modified FOLFIRI.3 vs modified FOLFOX as second-line therapy in patients with gemcitabine-refractory advanced pancreatic cancer. Br J Cancer. 2009;101(10):1658-63.

32. Berk V, Ozdemir N, Ozkan M, Aksoy S, Turan N, Inal A, et al. XELOX vs. FOLFOX4 as second line chemotherapy in advanced pancreatic cancer. Hepatogastroenterology. 2012;59(120):2635-9.

33. El-Hadaad HA, Wahba HA. Oxaliplatin plus 5-fluorouracil and folinic acid (OFF) in gemcitabine-pretreated advanced pancreatic cancer: a phase II study. J Gastrointest Cancer. 2013;44(3):313-7.

34. Trouilloud I, Dupont-Gossard AC, Malka D, Artru P, Gauthier M, Lecomte T, et al. Fixed-dose rate gemcitabine alone or alternating with FOLFIRI.3 (irinotecan, leucovorin and fluorouracil) in the first-line treatment of patients with metastatic pancreatic adenocarcinoma: an AGEO randomised phase II study (FIRGEM). Eur J Cancer. 2014;50(18):3116-24.

35. Sohal DPS, Kennedy EB, Khorana A, Copur MS, Crane CH, Garrido-Laguna I, et al. Metastatic pancreatic Cancer: ASCO clinical practice guideline update J Clin Oncol. 2018;36(24):2545-56

\section{Publisher's Note}

Springer Nature remains neutral with regard to jurisdictional claims in published maps and institutional affiliations.

Ready to submit your research? Choose BMC and benefit from:

- fast, convenient online submission

- thorough peer review by experienced researchers in your field

- rapid publication on acceptance

- support for research data, including large and complex data types

- gold Open Access which fosters wider collaboration and increased citations

- maximum visibility for your research: over $100 \mathrm{M}$ website views per year

At $\mathrm{BMC}$, research is always in progress.

Learn more biomedcentral.com/submissions 